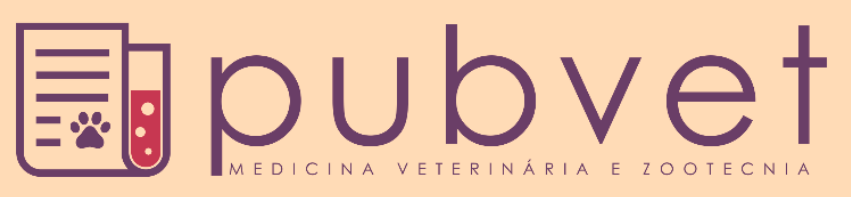

https://doi.org/10.31533/pubvet.v13n5a339.1-12

\title{
Manejo nutricional para cães e gatos obesos
}

\section{Lucas Pereira de Souza Silva ${ }^{1 *}$, Ronaldo César Hoog Nora Júnior ${ }^{1}$, Cinthia Maria Carlos Pereira $^{2}$, Verônica Maria Pereira Bernardino ${ }^{3}{ }^{\circ}$}

'Discentes do curso de Medicina Veterinária, Universidade Estadual de Santa Cruz (UESC), Ilhéus - Bahia, Brasil; ${ }^{2}$ Professora do Departamento de Ciências Agrárias e Ambientais, Universidade Estadual de Santa Cruz.(UESC), Ilhéus - Bahia, Brasil. ${ }^{3}$ Nutricionista Animal do Grupo FVO Brasília e Indústria e Comércio de Alimentos Ltda.

*Autor para correspondência: lucaspssouza3@gmail.com

Resumo. A obesidade é uma das desordens nutricionais mais frequentes em cães e gatos. A doença é caracterizada pelo acúmulo excessivo de gordura corporal de forma sistêmica, acarretando prejuízos secundários para a saúde do animal. Entre as causas da obesidade estão fatores como raça, espécie, pré-disposição genética, nutrição, castração, utilização de alguns medicamentos, mas também se deve à mudança no hábito de vida dessas espécies que ao passar do tempo foram tornando-se cada vez mais sedentárias. Por se tratar de um distúrbio nutricional, a adoção de um manejo nutricional é essencial no tratamento da doença, pois o balanceamento adequado de nutrientes como proteínas, lipídeos, carboidratos, fibras e a umidade do alimento, funcionará de forma reguladora no organismo do animal. Associado a um manejo nutricional adequado, a instituição de um programa de atividades físicas para reduzir o sedentarismo dos animais é de suma importância para auxiliar a perda de peso de maneira saudável e sem riscos à saúde do animal. Deste modo, pode-se afirmar que quando se fornece uma alimentação saudável e equilibrada a estes animais é a principal forma de controle da obesidade, portanto, é importante conhecer o papel de cada nutriente no tratamento, bem como a viabilidade da utilização de alimentos prontos disponíveis no mercado.

Palavras-chave: animal de estimação, doença, nutrição, tratamento

\section{Nutritional management for obese dogs and cats}

\begin{abstract}
Obesity is one of the most frequent nutritional disorders in dogs and cats. The disease is characterized by excessive accumulation of body fat in a systemic way, leading to secondary damage to the animal's health. Among the causes of obesity are factors such as race, species, genetic predisposition, nutrition, castration, use of some medications, but it is also due to the change in the habit of life of these species that over time were becoming more and more sedentary. Because it is a nutritional disorder, the adoption of nutritional management is essential in the treatment of the disease, because the proper balance of nutrients such as proteins, lipids, carbohydrates, fibers and food moisture, will function in a regulatory way in the animal's organism. Associated with an adequate nutritional management, the institution of a program of physical activities to reduce the sedentarism of the animals is of paramount importance to help the weight loss in a healthy way and without risks to the health of the animal. Thus, it can be said that providing a healthy and balanced diet to these animals is the main way to control obesity, so it is important to know the role of each nutrient in the treatment, as well as the feasibility of using ready-made foods available in the Marketplace.
\end{abstract}

Keywords: disease, nutrition, pet, treatment 


\section{Manejo nutricional para perros y gatos obesos}

Resumen. La obesidad es uno de los desórdenes nutricionales más frecuentes en perros y gatos. La enfermedad es caracterizada por la acumulación excesiva de grasa corporal de forma sistémica, acarreando en daños secundarios para la salud del animal. Entre las causas de la obesidad son factores como la raza, la especie, la predisposición genética, la nutrición, la castración, la utilización de algunos medicamentos, pero también se debe al cambio en el hábito de vida de esas especies que al paso del tiempo se fueron volviendo cada vez más sedentarias. Por tratarse de un trastorno nutricional, la adopción de un manejo nutricional es esencial en el tratamiento de la enfermedad, pues el equilibrio adecuado de nutrientes como proteínas, lípidos, carbohidratos, fibras y la humedad del alimento, funcionará de forma reguladora en el organismo del animal. Asociado a un manejo nutricional adecuado, la institución de un programa de actividades físicas para reducir el sedentarismo de los animales es de suma importancia para ayudar a la pérdida de peso de manera saludable y sin riesgos a la salud del animal. De este modo, se puede afirmar que proporcionar una alimentación sana y equilibrada a estos animales es la principal forma de control de la obesidad, por lo que es importante conocer el papel de cada nutriente en el tratamiento, así como la viabilidad del uso de alimentos listos disponibles en el tratamiento mercado.

Palabras clave: animal doméstico, enfermedad, nutrición, tratamiento

\section{Introdução}

A obesidade em cães e gatos, dentre os diversos conceitos, é mais bem descrita como uma patologia que tem como principal característica o acúmulo de gordura corporal, o que pode acarretar danos à saúde do animal (German, 2006), justificando assim a importância da realização de estudos acerca do assunto. Um motivo que reforça sua importância e risco é que anteriormente era relatada como uma doença em humanos, e atualmente já é uma realidade que afeta também os cães e gatos (Nascimento \& Amaral, 2010). A obesidade se tornou uma enfermidade de caráter epidêmico, visto que atinge os países subdesenvolvidos, quanto os desenvolvidos (Aptekmann et al., 2014b; Carciofi, 2005; Francischi et al., 2000). Linder \& Mueller (2014) demonstraram que cerca de 34 a $59 \%$ dos cães e 25 a $63 \%$ dos gatos apresentam-se obesos ou acima do peso.

Existem duas causas para o surgimento da obesidade: as naturais e as adquiridas. As principais causas naturais são fatores genéticos, raças, idade e alterações hormonais e as causas adquiridas são sedentarismo/falta de atividade física, castração, utilização medicamentosa e consumo irresponsável de alimento (Carciofi, 2005). Além disso, um ponto que deve ser destacado é a importância do tutor na rotina dos animais, afinal, muito dos vícios adquiridos pelos "pets" são de responsabilidade dos mesmos (Aptekmann et al., 2014b). Além do tutor, outra variável importante não deve ser esquecida é o ambiente que o animal vive, pois, o espaço físico também interfere nos hábitos, tanto comportamental, quanto alimentar, propiciando à obesidade (Aptekmann et al., 2014a; Aptekmann et al., 2014b). Apesar de a obesidade estar associada ao manejo nutricional e a deposição anormal de gordura, se não tratada pode servir de "gatilho", favorecendo o aparecimento de enfermidades tidas como secundárias, desencadeando respostas no sistema imune, digestório, cardiovascular, respiratório e osteoarticular (Feitosa et al., 2015) que podem ocasionar diminuição da qualidade e expectativa de vida. Além disso, torna o animal mais susceptível a patologias de caráter infeccioso e em casos de procedimentos cirúrgicos, a obesidade age como complicador durante sua realização (Lazzarotto, 1999).

Dessa forma, devido à importância dessa doença, serão abordados nessa revisão bibliográfica os principais pontos sobre obesidade, tais como o tratamento e prevenção.

\section{Descrição da doença}

A obesidade é caracterizada pelo excesso de gordura, localizada em todo o corpo animal, capaz de alterar de forma negativa a saúde do mesmo (German, 2010). Normalmente em um quadro obesidade, a função endócrina do tecido adiposo apresenta-se alterada na produção ou regulação de adipocinas, que por sua vez apresenta efeitos prejudiciais à saúde e ao organismo do animal. Sendo assim, a obesidade pode ser considerada também como uma condição inflamatória crônica, pois pacientes com essa 
enfermidade apresentam mediadores inflamatórios como necrose tumoral alfa (TNF- $\alpha$ ) e interleucinas 6 (IL-6) em níveis aumentados, sendo que estes diminuem à medida em que o paciente perde peso (Linder \& Mueller, 2014). A obesidade apresenta duas formas para sua classificação: a obesidade hiperplásica, que é ocasionada pelo aumento do número de adipócitos, e a hipertrófica, que é provocada pelo aumento do tamanho dos adipócitos presentes no tecido adiposo (Guimarães \& Tudury, 2006). Vale ressaltar que o organismo é capaz de aumentar o número de adipócitos, mas não é possível que esse número seja reduzido (Dodson et al., 2010). A obesidade hiperplásica ocorre devido à hiperestimulação do tecido adiposo pelo excesso de gordura, sendo preferencialmente acometidos os animais em fase de crescimento (Pereira et al., 2003), enquanto que a hipertrófica ocorre preferencialmente na fase adulta (Guimarães \& Tudury, 2006).

Embora a detecção primária da obesidade seja de fácil percepção, ter uma informação precisa do grau em que o animal se encontra é muito mais complexo, inclusive sendo necessária a utilização da avaliação do escore corporal, palpação e ultrassonografia para auxiliar no diagnóstico. Em algumas situações, o animal pode apresentar um volume muito grande de tecido muscular, o que gera um diagnóstico equivocado em relação à obesidade (Nelson \& Couto, 2015).

Por conta dos níveis de obesidade, técnicas de avaliação da composição corporal, sejam elas direta ou indireta são de extrema necessidade (Munday, 1994). Dentre as técnicas para medição, a mais utilizada é o IMCC (Índice de Massa Corporal Canina), pois reduziu a subjetividade da técnica, deixando o diagnóstico mais preciso (Muller \& Maicon, 2008), enquanto o mais preciso para felinos é a técnica de absorciometria de raios-x de dupla energia (DEXA) (Borges et al., 2008; Buelund et al., 2011). Embora a DEXA seja o diagnóstico mais preciso, o seu alto custo e dificuldade na sua execução acabam tornando-o inviável, e por conta disto, utilizam-se métodos mais simples e baratos, mas não menos eficazes, como os métodos de Escore de Condição Corporal (ECC) e Índice Massa Corporal (IMC) (Butterwick, 2000; Kimberly et al., 2010).

Tendo em vista que a obesidade apresenta diferentes graus de evolução, torna-se indispensável à adoção de uma ferramenta que permita avaliar a condição corporal do animal, com o objetivo de evitar comprometimentos na fisiologia normal do organismo e alterações metabólicas. O ECC (Escore de condição corporal) é um método subjetivo que correlaciona a proporção de gordura corporal com uma escala numérica que varia de 1 a 5 ou 1 a 9, sendo que a última escala possibilita uma avaliação mais precisa (Rodrigues, 2011). Segundo a classificação, considera-se que os índices de 1 a 3 indicam que o animal está abaixo do peso, de 4 e 5 representam uma faixa de peso ideal, e de 6 a 9 indicam um quadro de sobrepeso e obesidade. Um animal apresenta um escore corporal adequado quando este apresenta costelas palpáveis sem excesso de gordura recobrindo-as, e ao ser visto de cima apresente formato de ampulheta (Rodrigues, 2011). Linder \& Mueller (2014) consideraram animais com excesso de peso, aqueles que apresentem aumento de 10 a $20 \%$ do seu peso corporal, e os obesos quando o aumento é registrado de $20 \%$ ou mais.

\section{Fatores de risco}

O excesso de peso adquirido pelo animal seja ele cão ou gato vai gerar um desequilíbrio orgânico, e em consequência a isso proporcionar diversos riscos à saúde do animal. Isso ocorre pelo fato da obesidade ser um distúrbio altamente predisponente a muitas outras patologias, tais como problemas do sistema locomotor e articulações, alterações cardiopulmonares e endócrinas, como a diabetes mellitus, além de tornar o animal mais susceptível às enfermidades de natureza infecciosas, o que pode aumentar os riscos durante procedimentos cirúrgicos (Lazzarotto, 1999).

Embora seja caracterizada por muitos como uma doença essencialmente nutricional, a obesidade em sua origem evidencia uma complexidade ainda maior por existirem fatores genéticos, sociais, culturais, metabólicos que influenciam no desenvolvimento da enfermidade (Carciofi, 2005). Fatores relacionados aos proprietários não devem ser descartados, pois os mesmos são identificados como fator de risco ao desenvolvimento da obesidade em cães e gatos (Bland et al., 2010; Courcier et al., 2010; Lund et al., 2006).

Os animais considerados entre meia idade e senis tendem a ter uma predisposição a tornarem-se obesos. Os animais à medida que envelhecem, normalmente diminuem o gasto de energia proveniente da atividade física, e em contrapartida a essa diminuição, há um descenso da massa magra e alteração 
do metabolismo basal (Guimarães \& Tudury, 2006). Outro motivo comum que possibilita o surgimento da obesidade é a castração e muitos estudiosos sugerem que tal efeito (castração x obesidade) ocorre devido à redução na taxa metabólica do animal, tanto em macho, quanto em fêmeas (Oliveira et al., 2010). A sua relação ocorre de forma distinta entre ambos, sendo que as fêmeas na ausência do hormônio sexual (estrógeno) tendem a desenvolver polifagia e consequentemente um balanço energético positivo. Já nos machos, a ausência do hormônio sexual costuma afetar negativamente sua atividade sexual, além de reduzir também suas atividades físicas (Silva et al., 2016). A senilidade, juntamente com a questão genética pode ser considerada como causas naturais e ambas manifestam respostas diferentes em cada animal. Atrelado a isso, o cruzamento entre as diversas raças existentes de cada espécie (cão e gato) impossibilita uma precisão no efeito que pode ocorrer, visto que ainda assim, cada animal tende a desenvolver uma resposta distinta à um quadro de obesidade. Por tais motivos, a compreensão dos cruzamentos, porte físico e particularidades das raças se tornam importante para que o tutor diminua os riscos do surgimento ou agravamento do quadro de excesso de peso (Nelson \& Couto, 2015).

Os distúrbios endócrinos (Hipotireoidismo, Hiperadrenocorticismo e Hiperinsulinemia), embora correspondam apenas a 5\% da população de cães e gatos obesos, exigem cuidados, pois representam riscos para os animais com tais alterações. O hipotireoidismo vai acabar causando um descenso do metabolismo basal, predispondo a obesidade. Já no caso do hiper-adrenocorticismo, pode haver o aumento do seu peso corpóreo e consequentemente, pode haver elevação da massa corpórea do animal, já que há a utilização medicamentosa, principalmente corticosteroides sintetizados (Guimarães \& Tudury, 2006). No caso da hiper-insulinemia, vai estar associada ao aumento da pressão arterial como consequência de sua ação trófica na musculatura do vaso sanguíneo. Tal situação favorece a pressão arterial, ligada ao quadro de obesidade. $\mathrm{O}$ uso de determinados medicamentos, como anticonvulsivantes e glicocorticoides, pode causar ao animal polifagia, ou seja, aumento indiscriminado na ingestão de alimento (German, 2010). A obesidade ainda possibilita ao animal desenvolver problemas locomotores e enfermidades ortopédicas (Carciofi et al., 2005). A grande questão da obesidade em um quadro de alterações locomotora é justamente por possibilitar o surgimento de um ciclo de causa $\mathrm{x}$ efeito explicado por uma relação entre as duas alterações (obesidade - sistema locomotor), pois o excesso de peso tende a causar o aparecimento de artrite, devido à sobrecarga de peso nos membros do animal. Em consequência ao aumento do peso do animal e dores nas articulações há uma redução natural da atividade física e mobilidade, o que vai gerar um balanço energético positivo (caso não haja um cuidado do tutor com a dieta do animal obeso) que gera o aumento do peso do animal, agravando o quadro de artrite (Rodrigues, 2011).

A ocorrência de lesões no hipotálamo pode produzir a chamada obesidade hipotalâmica por desencadear alterações metabólicas (prejuízos da Termogênese, Hiperfagia, Hiperinsulinemia), que vão causar desordens na função do sistema nervoso autônomo (Pereira et al., 2003). As lesões do hipotálamo vão ocorrer de duas formas distintas, sendo a primeira de origem química, através de medicamentos, e a segunda de forma cirúrgica. Em ambas as situações, há uma alteração no metabolismo normal do animal, que produzirá o aumento da adiposidade (química). No caso das lesões cirúrgicas, a real noção da gravidade da lesão é de difícil identificação, visto que a área lesionada é delicada e na maioria das vezes não se sabe se o comprometimento foi parcial ou total da área (Pereira et al., 2003). Entre outros fatores, deve-se levar em consideração a interação gene nutriente, onde a composição nutricional da alimentação do animal pode desempenhar papel modulador de genes que determinam fenótipos dependentes de variação genética, principalmente a gordura. Dentre essas variações genéticas, a mais comum observada em relação à ocorrência da obesidade acontece com a IL-6, que é uma citocina próinflamatória, que apresenta o polimorfismo -174C/G (Steemburgo et al., 2009).

Baseando-se em um trabalho realizado nas cidades de Jaboticabal-SP e Viçosa-MG, o qual consistiu em entrevistas dos proprietários de cães atendidos pela Universidade Estadual Paulista "Júlio de Mesquita Filho" - Campus de Jaboticabal (UNESP-Jaboticabal) e Universidade Federal de Viçosa (UFV) respectivamente, para que fosse respondido um questionário com o total de 22 questões de múltipla escolha visando avaliar a noção dos tutores acerca do quadro dos seus animais. Após análise foi constatado que o principal causador da obesidade tem sido o excesso de alimentos (28\%), seguido pela baixa ou nenhuma atividade física (13\%). Surgiram outros motivos como castração (10\%), doenças (7\%), genética (7\%), e por fim, a utilização de medicamentos (4\%). Quando perguntado sobre o apetite 
dos seus animais, $48 \%$ responderam que acreditavam que seus animais se alimentavam normalmente, enquanto $49 \%$ responderam que o apetite era acima do normal e apenas $4 \%$ acreditavam que seus animais se alimentavam menos do que deveria. Mesmo não existindo uma comprovação científica nas respostas dos tutores, não há como negar que o aumento da ingestão de alimentos causa um balanço energético positivo, o que resulta no surgimento da obesidade, além de tornar transparentes os diversos motivos causadores ou potencializadores da obesidade (Aptekmann et al., 2014b).

\section{Manejo nutricional para cães e gatos obesos}

O tratamento da obesidade visa provocar redução do peso corporal dos animais. Para isso, recomenda-se a adoção de um manejo alimentar que forneça menor aporte calórico aliado à realização de exercícios físicos, levando o animal a um quadro balanço energético negativo (Carciofi, 2005; Carciofi et al., 2005). Entretanto, para instituir um manejo alimentar visando à perda de peso é de suma importância o levantamento do histórico completo do animal através de uma anamnese detalhada somada à realização de exames laboratoriais como urinálise, proteína total e fragmentada, perfil hepático, prova de função renal, hemograma, e, se possível, eletrocardiograma e radiografias, principalmente em animais idosos ou excessivamente obesos. Essas informações são importantes para a identificação do estado orgânico geral e endócrino do animal e para a avaliação de complicações patológicas secundárias que possam requerer adaptações específicas no programa de emagrecimento. Isto porque muitas vezes a consulta clínica não se deve ao fato do animal estar obesos, sendo assim, os animais diagnosticados obesos apresentam outras patologias associadas, devendo estas, ser tratado concomitantemente (Lazzarotto, 1999).

Os tutores desempenham papel fundamental durante o tratamento. Sendo assim, eles devem adotar a postura de não oferecerem ao animal nenhum tipo de alimento que não esteja dentro do planejamento estratégico e seguirem rigorosamente o plano de exercícios físicos, determinando assim o sucesso do tratamento (Mendes et al., 2013). Case et al. (2010) relataram que o manejo nutricional adequado deve apresentar um aporte calórico restrito. Desta forma, recomenda-se uma redução de $60 \%$ para cães e de 60 a $70 \%$ para gatos na quantidade de calorias fornecida na dieta, podendo estes valores serem alterados de acordo com o estado orgânico do paciente, evitando complicações secundárias à instituição de uma nova dieta. Sendo assim, com a adoção de restrição do aporte calórico na dieta objetiva-se uma redução de 1 a $2 \%$ do peso corporal para cães e 1,0 a 1,5\% para gatos por semana, pois, uma parte dos proprietários perde interesse pela utilização do programa de alimentação quando são instituídos objetivos de perdas menores devido a uma maior demora em observação de resultados. Neste contexto, uma redução de peso corporal semanal superior ao preconizado pode acarretar complicações secundárias como, por exemplo, a lipidose hepática felina, que ocorre devido ao catabolismo acelerado de gorduras. Para controle da eficiência do programa, deve reavaliar-se constantemente o peso do animal, alterando a dieta sempre que necessário, visando atender às exigências de cada etapa do tratamento. Espera-se então com o estabelecimento da restrição, uma redução de $15 \%$ do peso corporal dentro de 10 a 20 semanas (Freeman et al., 2011; Veiga, 2008).

Algumas modificações na composição da dieta são necessárias, tais como: aumentar a quantidade e melhorar qualidade das proteínas fornecidas, restringir os lipídeos e adequar à razão ômega 6:3, restringir a quantidade de carboidratos, aumentar a quantidade de fibras, aumentar a umidade da dieta e suplementar com L-carnitina. Essas ações visam promover um emagrecimento saudável do animal.

\section{Proteína}

A redução energética da dieta pode provocar uma diminuição da massa magra dos animais submetidos a um manejo nutricional com o objetivo de redução de peso para cães e gatos obesos, o que não é desejável. As proteínas auxiliam na manutenção de massa magra durante o processo de emagrecimento, gerando uma perda de peso com a redução da gordura corporal, e não pela perda de musculatura. Desta forma, preconiza-se então um grande aporte proteico com o objetivo de assegurar o fornecimento dos aminoácidos essenciais e não essenciais, e da energia necessária para a realização dos processos metabólicos. A utilização de proteínas na dieta de pacientes obesos torna-se ainda fundamental por gerarem maior incremento calórico durante a digestão, desta forma, os animais ao 
consumirem dietas ricas em proteínas, demandam de maior gasto energético para realizarem a digestão do alimento (Carciofi, 2007; Mendes et al., 2013).

Reduções drásticas no fornecimento de proteína, especialmente em gatos, podem levar ao desenvolvimento de um quadro de lipidose hepática, devido ao aumento do catabolismo de reservas corpóreas para geração de energia. Sendo assim, torna-se indispensável o fornecimento de proteínas de alto valor biológico tanto para gatos, quanto para cães, pois proporciona uma melhor condição corporal durante o emagrecimento. Gatos que receberam alimentação com $46 \%$ de proteína na matéria seca (MS) mostraram grande perda de gordura e maior manutenção de massa magra se comparado a gatos que consumiram alimentos com 36\%. Para cães uma dieta com 39\% de proteína na matéria seca demonstrouse com o melhor desempenho na manutenção da massa magra e perda de gordura (Case et al., 2010).

\section{Lipídeos}

Por serem os nutrientes com maior densidade calórica, os lipídeos são focos importantes de análise na instituição de um manejo nutricional. Lipídeos são facilmente armazenados em forma de gordura corporal como reserva energética, sendo assim, um fornecimento de uma dieta hiperlipídica leva a um acúmulo de tecido adiposo. A relação entre um alto consumo de lipídeos e obesidade pode ser explicada pelo fato de aumentarem a palatabilidade da dieta. Uma dieta com alto teor lipídico pode provocar alterações metabólicas como reduzir a secreção ou gerar resistência à função da leptina, hormônio produzido pelo tecido adiposo, que por mecanismos nervosos gera a sensação de saciedade (Pereira et al., 2003).

O consumo de uma dieta hiperlipídica pode ainda levar a um quadro de resistência insulinêmica, provocando um quadro de hiperinsulinemia. Esse, por sua vez, estimula uma secreção maior de amilina pelo pâncreas o que poderá gerar um acúmulo de amiloide nas células, dificultando a ação da insulina nestas, desenvolvendo um quadro de hiperinsulinemia associado com hiperglicemia e diabetes mellitus. Pode-se observar hipertrigliceridemia nesses casos, devido a um aumento de lipídios na circulação (Mendes et al., 2013). Para dietas com o objetivo de reduzir o peso deve-se então, restringir lipídeos na dieta na ordem de 6 a 11\% na matéria seca (Case et al., 2010).

No entanto, o fornecimento de lipídeos na dieta é importante, pois estes além de fornecer energia, são fontes de ácidos graxos essenciais. Estes ácidos são de suma importância para a homeostase do organismo na renovação de células, para o sistema imune, na função reprodutiva, etc. Os ácidos considerados essenciais são o ácido araquidônico (ômega 6), metabolicamente essencial, e os ácidos linoleico (ômega 6) e linolênico (ômega 3), que são nutricionalmente essenciais (Martin et al., 2006; Vianni \& Braz Filho, 1996). A relação ômega 6 e ômega 3 fornecida deve seguir a proporção de 5:1, tanto para cães, quanto para gatos, pois a obesidade trata-se também de uma enfermidade inflamatória, desta forma, essa proporção contribui para a ação anti-inflamatório do ômega 3 (Veiga, 2008).

\section{Carboidratos}

A utilização de carboidratos na dieta de gatos possui menor importância, pois, o suprimento energético é majoritariamente fornecido pela ingestão de proteínas. Além disso, gatos por serem carnívoros estritos, não são capazes de metabolizar grandes quantidades de carboidratos. Contudo, cães são capazes de realizar o aproveitamento de carboidratos, isto porque o seu processo de domesticação fez com que a espécie tivesse uma alimentação de origem animal e vegetal. Sendo assim, os carboidratos podem ser aproveitados pelos cães, porém, a ingestão na forma simples pode gerar altos picos glicêmicos, predispondo os animais à obesidade. Além disso, por serem mais facilmente digeridos e absorvidos, geram uma sensação de saciedade mais curta (Carciofi, 2008b; Veiga, 2008). Dessa forma, o fornecimento de carboidratos complexos em substituição a parte da gordura é uma boa opção, visto que possuem menos da metade da densidade calórica. Além de similar às proteínas, geram alto incremento calórico durante o processo digestivo, e diferentemente dos carboidratos simples. provocam uma digestão mais lenta, modulando a resposta glicêmica do organismo. Os exemplos de carboidratos complexos são o sorgo, lentilha, ervilha e a cevada (Case et al., 2010). Ao reduzir-se o fornecimento de carboidratos simples com a inclusão dos carboidratos complexos ocasiona uma absorção de glicose de forma mais lenta e, consequentemente menor liberação de insulina, que por sua vez é um hormônio que desempenha função lipogênica no fígado e nos adipócitos (Carvalheira et al., 2002). 


\section{Fibras}

Segundo Nelson \& Couto (2015), a perda de gordura é favorecida quando são adotadas dietas com baixo teor calórico e com a utilização de fibras que atuam reduzindo a sensação de fome, diminuindo o estresse da fome prolongado. Dessa forma, preconiza-se um balanço entre fibras solúveis e insolúveis na dieta, devido suas diferentes ações e funções.

As fibras solúveis utilizadas nas dietas possuem grande capacidade de reter água, retardar o esvaziamento gástrico e do trânsito do intestino delgado e afetarem negativamente a digestibilidade dos alimentos, pois, quando presentes no intestino formam um gel que impossibilita a ação de enzimas digestivas sobre o alimento ingerido. No entanto, durante a digestão intestinal, a fermentação das fibras solúveis culmina com a formação de ácidos graxos de cadeia curta, que se constituem em fonte de energia para o epitélio intestinal e para a microbiota local. A utilização destas fibras reduz a ocorrência de hiperglicemia pós-prandial, visto que provoca lentidão na digestão e absorção de carboidratos disponíveis na dieta, além de reduzir a lipidemia (Case et al., 2010).

As fibras insolúveis, não são fermentáveis e gelificantes, e possuem baixa capacidade de reter água, sua introdução na dieta é justificada pelo fato da taxa de passagem do alimento pelo trato digestivo ser rápida o que acarreta redução da absorção de nutrientes, devido o menor tempo de contato da digesta e o epitélio absortivo do trato gastrointestinal. Sua utilização, entretanto, pode aumentar o volume de massa fecal, devido a um menor aproveitamento dos alimentos ingeridos (Case et al., 2010).

\section{Umidade}

A umidade pode ser utilizada como uma ferramenta para diminuir a densidade calórica da dieta. Linder \& Mueller (2014) mostraram que gatos alimentados com dietas contendo maior teor de água na composição apresentaram redução no consumo voluntário de alimento, estando essa redução diretamente relacionada com a perda de peso em um curto tempo. No entanto, a opção por uma dieta mais úmida pode elevar o custo de alimentação do animal a depender do método adotado, já que alimentos comerciais enlatados com maior teor de umidade são mais caros.

\section{L-carnitina}

A L-carnitina é uma substância produzida no fígado e rins dos animais a partir da metionina e da lisina, dois aminoácidos essenciais. Possui importante função no transporte de ácidos graxos para o interior das mitocôndrias onde serão convertidos em energia, prevenindo que estes se sejam depositados no tecido adiposo (Case et al., 2010; Mendes et al., 2013). Recomenda-se a suplementação com Lcarnitina, pois pode auxiliar na manutenção de massa magra durante o manejo nutricional de animais obesos em emagrecimento. Por desempenharem função importante na oxidação de gorduras, estima-se que sua deficiência altere esse processo, resultando em acúmulo de gordura (Case et al., 2010; German, 2010).

\section{Frequência alimentar}

A frequência alimentar é definida como a quantidade de vezes em que o animal irá se alimentar durante o dia. Recomenda-se, um planejamento alimentar para redução de peso, o fornecimento de várias refeições durante o dia com quantidades pequenas, pois dessa forma, induz uma maior perda calórica por meio da termogênese decorrente do processo de digestão (Veiga, 2008). Segundo Aptekmann et al. (2014b) cães alimentados duas vezes ao dia, apresentaram maior sobrepeso em comparação a animais que tiveram a alimentação fracionada em mais vezes durante o dia. Veiga (2008) apresenta que a sensação de saciedade dos gatos está intimamente associada com o preenchimento do estômago, sendo assim, recomenda-se que animais obesos tenham sua alimentação dividida em várias refeições durante o dia de acordo com a disponibilidade do tutor e com a espécie, podendo variar de 3 a 6 vezes ao dia.

\section{Atividade física}

O sucesso de um planejamento alimentar envolve a menor ingestão calórica, através do fornecimento de alimentos com uma menor densidade energética, mas também do maior gasto calórico, seja por processos endógenos ou induzido através de exercícios físicos. Segundo estudos, observa-se um 
aumento do consumo em animais sedentários, o que ocasiona maior ganho de peso, pois a fisiologia normal parece não funcionar corretamente em indivíduos que estejam abaixo de uma faixa mínima de exercício físico. Nesses animais, a inclusão de quaisquer atividades físicas já pode ser capaz de gerar efeitos benéficos (Case et al., 2010). Para animais sedentários ou que não estejam acostumados, a inclusão de atividades físicas deve ser gradativa, evitando um esforço exagerado e uma sobrecarga de exercícios logo nos primeiros dias. Para cães, podem-se adotar caminhadas diárias de cerca de 20 minutos, a depender do estado sanitário do animal. As corridas, introdução de obstáculos no seu trajeto, atividades como nadar e brincar também faz parte do programa (Ribeiro \& Souza, 2017).

Com gatos, deve-se sempre estimulá-lo a se movimentar, caminhar, pular etc. devendo o tutor ser paciente, pois gatos são animais que costumam passar a maior parte do dia inativo e dormindo, portanto, essa mudança no comportamento deve ser realizada gradativamente, evitando-se a ocorrência de estresse (Mendes et al., 2013). Algumas práticas envolvem a utilização de brinquedos, o posicionamento estratégico das fontes de água e alimento fazendo com que se movimente. É possível colocar o alimento em locais mais altos induzindo o animal a pular ou subir escadas. Essas adaptações devem ser realizadas de acordo com o grau de obesidade e com a mobilidade do animal.

\section{Manutenção da perda de peso}

Uma vez instituído um manejo para perda de peso com sucesso, alguns animais requerem acompanhamentos contínuos com o objetivo de controlar a condição corporal e ingestão calórica. Recomenda-se a utilização de dietas balanceadas contendo boa proporção de nutrientes e calorias para atender a demanda energética necessárias para a manutenção do peso alvo (Linder \& Mueller, 2014). Esse monitoramento torna-se ainda mais importante quando é levado em consideração o fato de que pacientes que já foram obesos ou acima do peso apresentem maior predisposição e facilidade de ganharem peso em excesso novamente (Linder \& Mueller, 2014). Somado ao monitoramento da condição corporal do animal, recomenda-se a manutenção do plano de exercícios físicos para evitar acúmulos de gordura. Os petiscos e restos da alimentação humana devem ser evitados, pois alguns animais possuem tendência de ganharam peso mais rapidamente quando ingerem esse tipo de alimento (Case et al., 2010).

\section{Rações terapêuticas comerciais}

Sabe-se que muitos tutores buscam por uma forma mais prática e fácil de alimentar os animais de companhia. Sendo assim, em casos de cães e gatos obesos, uma das opções é realizar o tratamento com rações terapêuticas disponíveis no mercado. Para avaliar a viabilidade, a partir da composição nutricional, foram selecionadas três marcas aleatórias de rações terapêuticas para cães e para gatos obesos, e três rações para cães e para gatos em manutenção. As médias da quantidade de cada nutriente estudado são descritas na Tabela 1 e na Tabela 2.

As proteínas desempenham uma função de suma importância no manejo dietético de cães obesos, pois auxilia no emagrecimento saudável, uma vez que é fonte de aminoácidos essenciais e não essenciais; participa do processo de produção de energia para estes animai; compõem a estrutura da musculatura esquelética; além de realizarem incremento calórico no momento da digestão, demandando maior gasto energético do organismo neste processo. Sendo assim, pode-se observar na Tabela 1, uma maior quantidade de proteínas (33\%) nas rações terapêuticas em relação com as rações para animais em manutenção $(28,5 \%)$, justamente com o intuito de fornecer esse maior aporte proteico aos cães alimentados com este tipo de ração (Carciofi, 2007; Mendes et al., 2013).

Tabela 1. Comparação da composição de rações na matéria seca (MS) para cães obesos e cães em manutenção

\begin{tabular}{lcc}
\hline Componentes $^{1}$ & Rações Terapêuticas & Rações Manutenção \\
\hline Proteína Bruta (PB) & $33 \%$ & $28,5 \%$ \\
Extrato Etéreo (EE) & $8,4 \%$ & $13,7 \%$ \\
Extrato Não Nitrogenado (ENN) & $34,1 \%$ & $40,6 \%$ \\
Matéria Fibrosa & $11,6 \%$ & $4,3 \%$ \\
Energia Metabolizável & $3608 \mathrm{kcal} / \mathrm{kg}$ & $4197,7 \mathrm{kcal} / \mathrm{kg}$ \\
\hline
\end{tabular}

1Valores baseados nas rações comerciais terapêuticas e manutenção disponíveis no mercado. 
Os lipídeos encontram-se em menor quantidade nas rações terapêuticas, que apresentam média de $8,4 \%$ de extrato etéreo em sua composição em comparação com as rações para animais em manutenção que possuem em média 13,7\%. Essa redução ocorre devido à densidade energética dos lipídeos, pois, no manejo dietético para cães obesos, o que se deseja é reduzir essa carga energética diária proveniente da alimentação (Pereira et al., 2003).

Os cães por convenção são considerados animais onívoros, portanto, sua domesticação interferiu na alimentação fazendo com que sejam capazes de aproveitar os carboidratos de uma melhor forma em relação aos gatos. Porém, o fornecimento de carboidratos em grandes quantidades pode gerar picos glicêmicos e predispor os animais ao acúmulo de gordura, portanto, preconiza-se sua redução na dieta. Sendo assim, pode-se observar que as rações terapêuticas atendem à recomendação ao apresentarem menor quantidade de carboidratos em sua composição em relação às rações de manutenção, contendo $34,1 \%$ e 40,6 \% respectivamente em suas formulações (Carciofi, 2008a, 2008b; Veiga, 2008). Entretanto, a utilização de fontes de carboidratos complexos é desejável para animais nesta condição, pois além de fornecer maior incremento calórico na digestão, modula a curva de resposta glicêmica (Case et al., 2010).

Outro nutriente importante no manejo dietético de animais obesos é a fibra. Pode-se observar na Tabela 1, uma maior quantidade de fibras na ração terapêutica $(11,6 \%)$ em relação às rações para animais em manutenção $(4,3 \%)$, o que é desejável para a dieta destes animais. O fornecimento de um alimento que possua uma boa relação de fibras solúveis e insolúveis pode gerar efeitos desejáveis no trato gastrointestinal destes animais, pois atuarão reduzindo a digestibilidade e absorção de nutrientes da ingesta, levando também a um menor aproveitamento das calorias do alimento ingerido, o que pode contribui para a perda de peso (Case et al., 2010).

A ração terapêutica fornece uma menor quantidade de energia metabolizável em relação ao alimento destinado a animais em manutenção contendo $3608 \mathrm{kcal} / \mathrm{kg}$ e $4197,7 \mathrm{kcal} / \mathrm{kg}$ respectivamente, fornecendo menor aporte calórico aos cães alimentados com rações específicas para animais obesos (Carciofi, 2005; Carciofi et al., 2005).

Os gatos, por sua vez, também necessitam de um manejo nutricional criterioso durante o tratamento da obesidade, sendo assim, a análise da composição de alimentos comerciais para esta espécie também é importante para o fornecimento adequado de cada nutriente. Na Tabela 2 são listados os principais nutrientes da dieta, e feita uma comparação entre alimentos específicos para gatos obesos e para gatos em manutenção.

Tabela 2. Comparação da composição de rações na matéria seca (MS) para gatos obesos e para gatos em manutenção.

\begin{tabular}{lcc}
\hline Componentes $^{1}$ & Rações Terapêuticas & Rações Manutenção \\
\hline Proteína Bruta (PB) & $45,73 \%$ & $34,8 \%$ \\
Extrato Etéreo (EE) & $9,9 \%$ & $17,6 \%$ \\
Extrato Não Nitrogenado (ENN) & $23,1 \%$ & $33,1 \%$ \\
Matéria Fibrosa & $11,7 \%$ & $4,7 \%$ \\
Energia Metabolizável & $3662,1 \mathrm{kcal} / \mathrm{kg}$ & $4321,9 \mathrm{kcal} / \mathrm{kg}$ \\
\hline
\end{tabular}

1Valores baseados nas rações comerciais terapêuticas e manutenção disponíveis no mercado

Como apresentado anteriormente, o fornecimento da dieta para redução de peso através da degradação de gordura, pode ocasionar uma redução na massa muscular magra dos animais, o que é indesejável. Sendo assim, torna-se de suma importância o fornecimento de um maior aporte proteico que além de manter o funcionamento fisiológico energético normal no organismo do animal, favoreça a manutenção da massa magra, desta forma, pode ser observado um maior fornecimento deste nutriente nas rações terapêuticas $(45,73 \%)$ em relação aos alimentos para animais em manutenção $(34,8 \%)$ (Carciofi, 2007; Mendes et al., 2013). Pode-se observar uma redução no fornecimento de lipídeos na ração terapêutica para gatos obesos $(9,9 \%)$ em relação à ração para gatos em manutenção $(17,6 \%)$, isso se dá pelo fato de os lipídeos possuírem maior densidade calórica, portanto, considera-se prudente diminuir sua oferta em alimentos destinados à redução de peso, uma vez que a redução das calorias ingeridas é uma das metas terapêuticas (Pereira et al., 2003). A redução no fornecimento de ingredientes que apresentem altos níveos de carboidratos para pacientes obesos é uma das metas à serem alcançadas, 
observa-se que o alimento terapêutico apresenta menor índice de carboidratos na sua composição, sendo 23,1\% para este tipo de alimento contra 33,1\% na dieta para animais em manutenção (Carciofi, 2008a, 2008b; Veiga, 2008).

O maior índice de matéria fibrosa na ração terapêutica $(11,7 \%)$ em relação à ração para animais em manutenção $(4,7 \%)$ também atende às metas preconizadas para este nutriente no manejo do gato obeso, pois irão auxiliar na modulação da função do trato gastrointestinal (Case et al., 2010).

Uma das principais premissas no manejo dietético de animais obesos é a redução na ingestão calórica por parte dos animais, visando um balanço energético negativo e consequente perda de peso. Sendo assim, na análise das rações comerciais disponíveis no mercado pode-se observar uma redução na energia metabolizável do alimento terapêutico que apresentou média de $3662,1 \mathrm{kcal} / \mathrm{kg}$ em relação ao alimento para animais em manutenção que apresentou $4321,9 \mathrm{kcal} / \mathrm{kg}$. Esta redução tem como objetivo fornecer menos energia ao animal, evitando que haja excessos que porventura se acumulem no corpo em forma de gordura (Carciofi, 2005; Carciofi et al., 2005).

\section{Considerações finais}

Pode-se concluir que a adoção de um programa de exercícios somado à uma alimentação criteriosa são de suma importância no tratamento da obesidade, pois o gasto energético proveniente dos exercícios físicos e o balanceamento adequado dos principais nutrientes presentes na dieta de cães e gatos são capazes de provocar efeitos benéficos ao organismo do animal, auxiliando na redução do acúmulo e gordura e tratamento da doença.

\section{Referências bibliográficas}

Aptekmann, K. P., Mendes Junior, A. F., Passo, C. B., Secchin, M. C. \& Galeas, M. A. V. (2014a). Comparação dos diferentes métodos de avaliação corporal em felinos. Brazilian Journal of Veterinary Medicine, 36(2):215-218.

Aptekmann, K. P., Suhett, W. G., Junior, A. F. M., Souza, G. B., Tristão, A. P. P. A., Adams, F. K. \& Tinucci-Costa, M. (2014b). Aspectos nutricionais e ambientais da obesidade canina. Ciência Rural, 44(11):2039-2044.

Bland, I. M., Guthrie-Jones, A., Taylor, R. D. \& Hill, J. (2010). Dog obesity: veterinary practices' and owners' opinions on cause and management. Preventive Veterinary Medicine, 94(3-4):310-315.

Borges, N. C., Vasconcellos, R. S., Canola, J. C., Paula, F. J. A., Carciofi, A. C. \& Pereira, G. T. (2008). Precisão da técnica de absorciometria de raios-x de dupla energia na determinação da composição corporal em gatos. Arquivo Brasileiro de Medicina Veterinária e Zootecnia, 60263-266.

Buelund, L. E., Nielsen, D. H., Mcevoy, F. J., Svalastoga, E. L. \& Bjornvad, C. R. (2011). Measurement of body composition in cats using computed tomography and dual energy X-ray absorptiometry. Veterinary Radiology \& Ultrasound, 52(2):179-184.

Butterwick, R. (2000). How fat is that cat? Journal of Feline Medicine and Surgery, 2(2):91-94.

Carciofi, A. C. (2005). Obesidade e suas conseqüências metabólicas e inflamatórias em cães e gatos. Jaboticabal.

Carciofi, A. C. (2007). Métodos para estudo das respostas metabólicas de cães e gatos a diferentes alimentos. Revista Brasileira de Zootecnia, 36(Sup.):235-249.

Carciofi, A. C. (2008a). Fontes de proteína e carboidratos para cães e gatos. Revista Brasileira de Zootecnia, 37(Sup.):28-41.

Carciofi, A. C. (2008b). Manejo nutricional do cão e do gato hospitalizado. Paper presented at the Apontamentos teóricos das disciplinas de clínica das doenças carenciais, endócrinas e metabólicas e de nutrição e alimentação de cães e gatos, Jaboticabal.

Carciofi, A. C., Gonçalves, K. N. V., Vasconcellos, R. S., Bazolli, R. S., Brunetto, M. A. \& Prada, F. (2005). A weight loss protocol and owners participation in the treatment of canine obesity. Ciência Rural, 35(6):1331-1338. 
Carvalheira, J. B. C., Zecchin, H. G. \& Saad, M. J. A. (2002). Vias de sinalização da insulina. Arquivos Brasileiros de Endocrinologia \& Metabologia, 46(4):419-425.

Case, L. P., Daristotle, L., Hayek, M. G. \& Raasch, M. F. (2010). Canine and feline nutrition: A Resource for companion animal professionals: Elsevier Health Sciences.

Courcier, E. A., Thomson, R. M., Mellor, D. J. \& Yam, P. S. (2010). An epidemiological study of environmental factors associated with canine obesity. Journal of Small Animal Practice, 51(7):362367.

Dodson, M. V., Hausman, G. J., Guan, L., Du, M., Rasmussen, T. P., Poulos, S. P. \& Jiang, Z. (2010). Lipid metabolism, adipocyte depot physiology and utilization of meat animals as experimental models for metabolic research. International Journal of Biological Sciences, 6(7):691-699.

Feitosa, M. L., Zanini, S. F., de Sousa, D. R., Carraro, T. C. L. \& Colnago, L. G. (2015). Fontes amiláceas como estratégia alimentar de controle da obesidade em cães. Ciência Rural, 45(3):546551.

Francischi, R. P. P., Pereira, L. O., Freitas, C. S., Klopfer, M., Santos, R. C., Vieira, P. \& Lancha Junior, A. H. (2000). Obesidade: atualização sobre sua etiologia, morbidade e tratamento. Revista de Nutrição, 13(1):17-28.

Freeman, L., Becvarova, I., Cave, N., MacKay, C., Nguyen, P., Rama, B. \& Yathiraj, S. (2011). Nutritional Assessment Guidelines: information. Journal of the South African Veterinary Association, 82(4):254-263.

German, A. (2010). Obesity in companion animals. In Practice, 32(2):42-50.

German, A. J. (2006). The growing problem of obesity in dogs and cats. The Journal of Nutrition, 136(7):1940S-1946S.

Guimarães, A. L. N. \& Tudury, E. A. (2006). Etiologias, conseqüências e tratamentos de obesidades em cães e gatos-revisão. Veterinária Notícias, 12(1):29-41.

Kimberly, B., Bartges, J., Buffington, T., Freeman, L., Grabow, M. \& Legred, J. (2010). Nutrition assessment guidlines for dogs and cats. Journal American Animal Hospital Association, 26285-289.

Lazzarotto, J. J. (1999). Revisão de literarura: Relação entre aspectos nutricionais e obesidade em pequenos animais. Revista da Universidade de Alfenas, 533-35.

Linder, D. \& Mueller, M. (2014). Pet obesity management: beyond nutrition. Veterinary Clinics: Small Animal Practice, 44(4):789-806.

Lund, E. M., Armstrong, P. J., Kirk, C. A. \& Klausner, J. S. (2006). Prevalence and risk factors for obesity in adult dogs from private US veterinary practices. International Journal of Applied Research in Veterinary Medicine, 4(2):177-186.

Martin, C. A., Almeida, V. V. d., Ruiz, M. R., Visentainer, J. E. L., Matshushita, M., Souza, N. E. d. \& Visentainer, J. V. (2006). Omega-3 and omega-6 polyunsaturated fatty acids: importance and occurrence in foods. Revista de Nutrição, 19(6):761-770.

Mendes, F. F., Rodrigues, D. F., Prado, Y. C. L. \& Araújo, E. G. (2013). Obesidade felina. Enciclopédia Biosfera, 9(16):1602-1625.

Muller, J. D. C. M. \& Maicon, P. E. S. (2008). Adaptação do índice de massa corporal humano para cães. Ciência Rural, 38(4):1038-1043.

Munday, H. S. (1994). Assessment of body composition in cats and dogs. International Journal of Obesity, 1814-21.

Nascimento, B. C. L. \& Amaral, R. W. C. (2010). Obesidade em cães e seus efeitos em biomarcadores sanguíneos-revisão de literatura. PUBVET, 4Art. 795-801.

Nelson, R. W. \& Couto, C. G. (2015). Medicina interna de pequenos animais. Amsterdan: Elsevier Editora.

Oliveira, M. C., Nascimento, B. C. L. \& Amaral, R. W. C. (2010). Obesidade em cães e seus efeitos em biomarcadores sanguíneos-revisão de literatura. PUBVET, 4Art. 795-801. 
Pereira, L. O., Francischi, R. P. \& Lancha Júnior, A. H. (2003). Obesidade: hábitos nutricionais, sedentarismo e resistência à insulina. Arquivos Brasileiros de Endocrinologia \& Metabologia, 47(2):111-127.

Ribeiro, F. R. \& Souza, M. A. (2017). Aspectos nutricionais e ambientais na obesidade canina: estudo de caso. Políticas e Saúde Coletiva, 2(3):15-27.

Rodrigues, L. F. (2011). Métodos de avaliação da condição corporal em cães. Paper presented at the Universidade Federal de Goiás, Goiânia.

Silva, S. F., Brito, A. K. F., Freire, B. A. A., Sousa, L. M. \& Pereira, I. M. (2016). Obesidade canina: Revisão. PUBVET, 11(4):313-423.

Steemburgo, T., Azevedo, M. J. \& Martínez, J. A. (2009). Interação entre gene e nutriente e sua associação à obesidade e ao diabetes melito. Arquivos Brasileiros de Endocrinologia \& Metabologia, 53(5):497-508.

Veiga, A. P. M. (2008). Suscetibilidade a diabetes mellitus em cães obesos. Acta Scientiae Veterinariae, 36(3):311-312.

Vianni, R. \& Braz Filho, R. (1996). Ácidos graxos naturais: importância e ocorrência em alimentos. Química Nova, 19400-407.

Recebido: 24 de abril, 2019.

Aprovado: 17 de maio, 2019

Publicado: 8 de junho, 2019.

Licenciamento: Este artigo é publicado na modalidade Acesso Aberto sob a licença Creative Commons Atribuição 4.0 (CC-BY 4.0), a qual permite uso irrestrito, distribuição, reprodução em qualquer meio, desde que o autor e a fonte sejam devidamente creditados. 